\title{
Özgür Yazılım ve Açık Kaynağın Güncel Kullanımına Örnekler ve Durum İncelemesi
}

\author{
Elif AŞICI ${ }^{\mathrm{a} 1}$, Aydın Tarık ZENGINN ${ }^{\mathrm{b} 2^{*}}$ \\ a İstanbul Sabahattin Zaim Üniversitesi, Mühendislik ve Doğa Bilimleri Fakültesi, Bilgisayar Bilimi ve Mühendisliği Yüksek Lisans Programı, İstanbul, Türkiye \\ b İstanbul Sabahattin Zaim Üniversitesi, Mühendislik ve Doğa Bilimleri Fakültesi, Bilgisayar Mühendisliği, Istanbul, Türkiye
}

İstanbul Sabahattin Zaim Üniversitesi Fen Bilimleri Enstitüsü Dergisi (2021) 3 (1): $21-25$

https://doi.org/10.47769/izufbed.866262

(iD) ORCID ${ }_{1}$ 0000-0001-6956-7898; ${ }^{2}$ 0000-0002-0860-4509

\begin{tabular}{l}
\hline YAYIN BİLGİSİ \\
\hline Yayın geçmişi: \\
Gönderilen tarih: 1 Şubat 2021 \\
Kabul tarihi:14 Şubat 2021 \\
\end{tabular}

Anahtar kelimeler:

Açık kaynak

Açık kaynak yazılım

Açık kaynak donanım

OSS

FLOSS

\section{ÖZET}

Açık Kaynak ve Özgür Yazılımlar verimlilik, kullanılabilirlik, güvenlik, tasarruf, girişimcilik ve daha birçok açısından oldukça önemlidir. Bu çalışma, açık kaynak ve özgür yazılım başlığı altındaki "özgür yazılım", "açık kaynak yazılım" ve "açık kaynak donanım" kavramları ile ilgili farklı yazarlar tarafından elde edilen çalışmaların incelemesini sunmaktadır. Bu sayede çalışmalar hakkında okuyucuya özet bilgiler sunarak konu ile ilgili okuyucunun ön bilgilenmesini sağlamak ve gerekli olan yol haritasını ortaya koymayı hedeflemektedir. Bu çalı̧̧ma, genel olarak "Eğitim", "Sağlık", "Bilişim-Teknoloji" ve "Endüstri" alanındaki yayınlar üzerine yoğunlaşmıştır.

\section{Examples of Current Use of Free Software and Open Source and Case Study}

\begin{tabular}{l}
\hline ARTICLE INFO \\
\hline Article history: \\
Received: 1 February 2021 \\
Accepted: 14 February 2021
\end{tabular}

Key words:

Open source

Open source software

Open source hardware

OSS

FLOSS

\begin{abstract}
Open Source and Free Software are advantageous in terms of efficiency, usability, security, savings, entrepreneurship and many other aspects. In this article, under the title of open source and free software, it presents a review of the studies obtained by different authors on the concepts of "free software", "open source software" and "open source hardware". Thanks to this article, the reader has been provided with summary information about various studies, as well as providing the opportunity to obtain preliminary information about their field of study, aiming to reveal the road map that the reader will progress. This article focuses on studies in the fields of "Education", "Health", "Information-Technology" and "Industry" in general.
\end{abstract}

\section{Giriş}

Açık kaynak, bir bilgisayar yazılımının makine diline dönüştürülüp kullanımından önceki, programcılar tarafindan okunur, anlaşılır, yeni amaçlara uygun değiştirilebilir hâlinin gizli tutulmayıp açık, yani okunabilir hâlde kamuyla paylaşılıyor olmasına denilmektedir. Açık kaynak kodlu yazılımların da diğer yazılımlarda olduğu gibi lisanslarının olması gerekmektedir. Bu lisanslar, açık kaynak kodlu yazılımın kullanımı, dağıtımı, geliştirilmesi gibi yazılımın belli haklarını ve sınırlarını koruyan, yazılımı geliştiren ve kullanacak, değiştirecek, dağıtacak kişiler arasındaki kontratlardır. MIT LICENSE, APACHE LICENSE ve GNU vb birçok açık kaynak lisans1 bulunmakta olup doğru lisans seçimi büyük önem taşımaktadır (Kapitsaki ve Charalambous, 2016) (Lerner ve Tirole, 
2005). Açık kaynak yazılımlarının temelinde kullanılabilirlik, öğrenilebilirlik, verimlilik ve memnuniyet anahtar kelimeleri barınmaktadır. Açık kaynak yazılımların kabul edilebilirliğinde kullanılabilirlik, tasarım, geliştirici ve kullanıcı arasındaki iletişimin iyileştirilmesi gibi çeşitli unsurların etkili olduğu kanısı elde edilmiştir (Dawood ve diğerleri, 2019). Açık kaynak kodlu yazılımların kişi veya kurumlara güvenlik, ekonomi, kalite, kullanılabilirlik, uygulanabilirlik gibi birçok avantajlar sağladığı ve bu sayede sağllk, eğitim, endüstri, teknoloji ve çeşitli alanlarda gün geçtikçe kullanımının arttığı görülmektedir.

Bu çalışmada, özgür ve açık kaynak yazılımların kullanım alanları içerisinden incelemek üzere Eğitim, Endüstri, Sağlık ve Teknoloji alanları seçilmiştir. Özgür yazılım ve açık kaynağın güncel kullanımına örnekler verilmiş olup ilgili alanların durum incelemesi yapılmıştır. Çalışmada bölüm 2.1, 2.2, 2.3 ve 2.4'de sırası ile Eğitim, Endüstri, Sağlık ve Teknoloji alanları ele alınmıştır.

\section{Sistematik Literatür İncelemesi}

\subsection{Eğitim}

Eğitimde açı kaynaklı donanım ile ilgili sistematik bir haritalama çalışması yapılmıştır. Bu çalışmada, 2012'den beri açık kaynak donanımların eğitim ve araştırma alanlarında uyandırdığı büyük ilgi doğrultusunda yayın sayılarında büyük artış elde edildiği sonucuna varılmış ve 2014 'ten beri üniversite çalışmaları elektronik, otomasyon ve bilgisayar programlama alanlarında artış olduğu saptanmıştır (Heradio ve diğerleri, 2018). Eğitimin başlangıcında öğrencileri cesaretlendirmek ve onları Fen, Teknoloji, Mühendislik, Matematik (STEM) gibi alanlara teşvik etmek için açık kaynak platformlar kullanılmıştır (Yakimov, 2018). Ekip ve proje tabanlı çalışmalara daha çok üniversite ve kamu alanlarında rastlanmaktadır. Proje tabanlı öğrenmelerin (PBL) metodolojisi tek başına ekip çalışmasını sağlamadığından işbirliğine dayalı öğrenme (CL) ile tamamlanmış olup, oluşturulan bu yapı öğrencilere kurs olarak verilmiş ve gözlemlenmiştir. Bunun sonucunda, işbirliğine dayalı proje tabanlı öğrenmenin ve akran değerlendirmesi toplantı metodolojilerinin tanıtılmasının öğretme, öğrenme sürecini iyileștirdiği görülmüștür (Rodriguez-Sanchez, Torrado-Carvajal, Vaquero, Borromeo ve Hernandez-Tamames, 2016). Açık kaynaklı projeleri sınıf ortamına entegre etmek basit bir öneri değildir. Ancak sonuçları, ögrencilerin pratik deneyim kazanması açısından büyük bir firsat olarak görülmektedir (DeKoenigsberg, 2008). Örneğin yazılım mühendisliği dersinde açık kaynaklı projeleri kullanmak, modelleme, hataları çözme, yeni özellikler ekleme ve benzer bir çok işlemde öğrencilere avantaj sağlamaktadır (Dorodchi ve Dehbozorgi, 2016). Üniversitelerde müzik teorisi derslerini desteklemek adına Troubadour Platformu geliştirilmiştir. Bu platform kulak eğitimi için açık kaynaklı, kişiselleştirilmiş ve uyarlanabilir bir web platformdur. Uygulamaya geçirildiğinde, sınav performanslarında artış, öğrenci deneyimine destek ve öğreticilerin iş yükünde azalma sağladığı sonuçlarına varılmıştır (Pesek, Vucko, Savli, Kavcic ve Marolt, 2020). Son olarak Türkiye'de eğitim alanı içerisinde açık kaynak kavramını inceleyen makaleler ele alınmıștır. Konu ile ilgili ilk çalışma FATIH projesi ile birlikte okullarda kullanılmaya başlanan akıllı tahta sistemlerini etkin bir şekilde kullanan öğretmenlerin görüşlerini öğrenmek için yapılmıştır. FATİH projesinde, TÜBİTAK bünyesinde geliştirilen Pardus işletim sistemi kullanılmıştır. Bu sistemi kullanan öğretmenlerden sistem hakkındaki görüşleri toplanmış ve çeşitli analizler yapılmıştır. Öğretmenlerin pozitif ortak görüşleri yerli ve milli kaynak olan Pardus işletim sistemini kullanıyor olmalarını destekledikleri yönünde olmuştur. Bu görüşe ek olarak güvenlik, EBA'ya doğrudan erişim imkânı, hızlı ve kolay kullanım, kişiselleştirilebilir masaüstü imkanı ve düşük maliyetli oluşu FATİH projesini destekleyen diğer görüşler arasında sıklıkla yer almıştır (Cantürk, 2020). İncelenilen diğer çalışmada, eğitim alanında hayata geçirilen FATIHH projesi kapsamında öğrencilerin teknolojik yetersizliklere rağmen düşük maliyetli elde edilebilen cihazlar sayesinde her öğrenim seviyesine uygun bir şekilde tasarlanarak kaliteli bilgiye ulaşabilmesi hedeflenmiş olup bu kapsamda çevre ülkelere bir örnek teşkil etmiş̧ir (Milla, Kurt ve Mataruna-Dossantos, 2019). Genel bir perspektifte değerlendirecek olursak eğitim alanında kullanılan açık kaynak kavramı donanımsal ve yazılımsal anlamda öğrencilere, akademisyenlere ve farklı birçok gruba kullanılabilirlik, düşük maliyet ve kalite başta olmak üzere pek çok alanda avantaj sağlamaktadır.

\subsection{Endüstri}

Açık kaynak ve Özgür Yazılımlar bir çok alanda her geçen gün daha fazla tercih edilmektedir. Otomotiv, robotik, yapay zeka, haberleşme endüstrisi olmak üzere karşımıza çeşitli alanlarda çıkmaktadır. Bunun sebebi düşük maliyet, güvenilirlik, kullanılabilirlik ve daha birçok faktör olabilir. Bahsedilen bu bilgiler doğrultusunda incelediğimiz bu makalede, bilgi teknolojileri (IT) alanında kurum, kuruluşların özgür ve açık kaynak kodlu yazılımı (FLOSS) benimsemesi ve benimsenme süresini etkileyen faktörler ele alınıp bu konu üzerine bir kılavuz oluşturulmuştur. FLOSS un benimsenmesini etkileyen faktörler: teknolojik, organizasyonel ve ekonomik faktörler olarak belirtilmiştir (Sánchez, Ayuso, Galindo ve Benavides, 2020). Yazılım projelerindeki değişikliklerin ve süreç iyileştirme stratejilerinin maliyet etkinliğini değerlendirilmesi için, açık kaynak yazılım olan Spring Framework ile bir süreç değerlendirmesi yapmıştır. Bunun sonucunda, simülasyon modelleri, değişikliklerin etkisini değerlendirerek ve sürecin dinamik görünümlerini görselleştirerek fikir birliği oluşturmayı desteklemek için etkili bir araç olarak kullanılabileceği kanısına varılmıştır (Zhang, Wang ve Kang, 2018).

Maliyet açısından detaylı inceleme yapılacak olursa, düşük maliyetli 3D Metal Yazıcı açık kaynaklı bir mikrodenetleyici ile kontrol edilmiş olup düşük maliyetli bir ticari kaynak makinesi ve deltabot RepRap olan Rostock'un bir türevinin kombinasyonu şeklinde üretilmiştir (Anzalone, Zhang, Wijnen, Sanders ve Pearce, 2013). Maliyet avantajını konu alan başka bir makale incelendiğinde, CAD yazılımında tasarlanan sanal bir modelden üç boyutlu fiziksel modeller üreten bir 3D yazıcının tasarımınının ve yapımının işlendiği görülmektedir (Acuna, Rivas, Chancusi ve Navarrete, 2015). Çalışmalar sonucunda: prototipleme, düşük maliyetli plastik parçalar ve ücretsiz yazılım kullanarak bir 3D yazıcının tasarlanmasının ve oluşturulmasının mümkün olduğu belirtilmiştir (Acuna ve diğerleri, 2015; Anzalone ve diğerleri, 2013).

Maliyetin ve sürdürülebilirliğin önemli olduğu enerji alanında açık kaynak yazılım-donanım sayesinde çeşitli prototipler üretilerek çeşitli testler yapılmıştır. İncelenen ilk çalışmada, büyük miktarda yenilenebilir enerjinin entegrasyonu, enerjiyi uzun mesafelerde güvenilir bir şekilde taşımak için çok terminalli yüksek voltajlı doğru akım (MTDC) şebekeleri gerektiği düşünülmüştür. Bu yüzden çalışmada açık kaynaklı, düşük maliyetli ve korumalı akıllı elektronik cihaz (IED) prototipi incelenilerek IED'nin bu kurulumda MTDC şebeke koruması için gerekli hıza ulaştığını gösteren simüle edilmiş iki arıza durumu için örnek sonuçlar sunulmuştur (Jahn, Hohn, Chaffey ve Norrga, 2020). Ölçeklenebilirlik, bulut bilişimde potansiyel sorun haline geldiği dönemde, sis kompozit enerji yönetimi için yeni bir platform tanıtılmıştır. Açık kaynak yazılım ve donanımın özelleştirilebilir yeteneği, kullanıcının kontrollü enerji yönetimi platformuna bir hizmet olarak eklemesini sağlarken, uygulama maliyeti ve pazara sunma sürelerini önemli ölçüde azaltacaktır (Al Faruque ve Vatanparvar, 2016).

Sistemsel olarak açık kaynaklar ele alındığında sistemin devamlılığını incelemek ve çalışmamızın seyri adına çeşitli makaleler incelenmiștir. Kurumlarda, okullarda vs. kullanılan yazılımlardaki sürümler arasında sorunların çözülmesini anlamak adına açık kaynak yazılım için homojen olmayan Poisson Süreci modeli geliştirilmiştir. Önerilen modelden tahmin edilen optimum yayın süresi, farklı aktif kullanıcı memnuniyet seviyelerinde gözlemlenen yayın süresine yakındır. Önerilen karar modeli, yönetimin en uygun yayın-güncelleme zamanını doğru şekilde belirlemesine yardımcı olabileceği belirtilmiștir (Singh, Sharma ve Pham, 2018). Diğer bir önemli açık kaynak projesi üzerinde mimari 
dokümantasyonun etkisi incelenmek istenmiş ve geniş çaplı bir inceleme yapıldığında, çekirdek proje üyelerinin mimari endişelerini ve çözümlerini kaydetmede önemli bir amaca hizmet ettiği sonucu elde edilirken tüm proje üyelerine eşit derecede iyi hizmet vermediği belirlenmiştir (Kazman, Goldenson, Monarch, Nichols ve Valetto, 2016). Üyelerin deneyimleri ve çalışma yıllarına göre proje üzerindeki etkileri değişiklik gösterebilir. Projenin başarısını etkileyecek unsurlardan biride, zor ve kapsamlı projelere sonradan katılan ekip çalışanlarıdır. Bu çalışmada, FLOSS projelerinin başarılı olabilmesi için kritik projelere sonradan dahil olanların sorun izleme sistemine (ITS) katılmasının ve ortamda uzun vadede katkıda bulunanlardan (LTC) olma olasılıklarını nasıl etkilediğini ölçmek adına bir çalışma yapılmıştır. Sonuçlar incelendiğinde, ilk ayda en az bir sorunun düzeltilmesini bildirebilen yeni gelenlerin, LTC olma ihtimalinin diğerlerine göre iki kat daha fazla olduğu görülmüştür (Zhou ve Mockus, 2015). FLOSS topluluğu için dönemsel gönüllülük (EV) kavramı incelendiğinde, gönüllü kişilerin motivasyonu, sosyal normlar, psikolojik topluluk duygusu, tatmin ve topluluk bağlılığ şeklindeki 5 kavramın EV için önemli faktörler olduğuna ve EVinde FLOSS'ta yaygın olduğu kanısına varılmıştır (Barcomb, Kaufmann, Riehle, Stol ve Fitzgerald, 2018).

Kamuda açık kaynak yazılım sistemlerinin değerlendirilmesi için bir çalışma yapılmıştır. Açık kaynak yazılım ekosisteminin (OSSE) ilerleyişinin, geliştiricilerin kullanıcı geri bildirimlerini ve sistem parametrelerini toplayarak bir OSSE nin kendi durum değerlendirmesini yapmalarına yardımcı olabilecek yarı niceliksel yöntemlerle ölçülebileceği sonucuna varılmıștır (Wang, Wan ve Gao, 2019).

\subsection{Sağlık}

Sağlık alanında yazılımdan donanıma kadar bir çok uygulama, proje ve sistem bileşenlerinde Açık Kaynak kullanımı mevcuttur. Açık kaynak yazılımlarda Modelleme yöntemleri kullanılarak, daha geniş bir araştırmacı kitlesinin erişimine imkan sağlamak için açık kaynak olan OpenSim'de altı serbestlik dereceli tibiofemoral ve bir serbestlik dereceli patellofemoral eklemi oluşturulmuştur (Schmitz ve Piovesan, 2016). OpenSim'de olușturulan bir diğer model ise, doğru yürüyüş simülasyonları oluşturmak için kullanılabilecek sağlıklı genç bireylerin açık kaynaklı, üç boyutlu bir kas-iskelet modelidir (Rajagopal ve diğerleri, 2016). İlgili bu makalelerde (Rajagopal ve diğerleri, 2016; Schmitz ve Piovesan, 2016), geniş bir kitleye erişilebilir bir araç olarak modelleme sunulmuş oldu.

Mevcut araştırmaları kolaylaştırmak adına işitme sağlı̆̆ hizmetlerinde modern ve açık kaynaklı bir işitme araştırma platformu olan Açık Konuşma Platformu (OSP) geliştirilmiştir (Pisha ve diğerleri, 2019). Konuşma işleme algoritmaları, insan sesi algıs1, işitme cihazı uydurma prosedürleri gibi özelliklere sahip bu set daha önce hiç mümkün olmayan yeni araştırma türlerine olanak sağlamak için tasarlanmıştır. Sağlık alanında açık kaynakların kazandırdığı avantajlardan bir tanesi de zaman avantajıdır. Bir kişiden nörofizyolojik deneyler için Elektroensefalogram (EEG) sinyallerini edinmesine, görselleştirilmesine, filtrelemesine, işlemesine ve beyin bilgisayar arayüzü (BCI) deneylerinin gerçekleştirilmesine olanak tanıyan açık kaynaklı bir BCI platformu sunulmuştur (Chiesi, Guermandi, Placati, Scarselli ve Guerrieri, 2019). Bahsedilen konu doğrultusunda incelediğimiz bu çalışmada, platformun işlemler için gerekli zamanı azalttığ 1 sonucuna varılmıştır.

Günümüzde giyilebilir ve taşınabilir şekilde üretilen cihazlar yardımı sayesinde, kullanıcılardan çeşitli veriler elde edilmektedir. Bahsedilen konu ile ilgili bir çalışmada, giyilebilir cihazlar ve vücut sensörü ağları için deneysel verilere erişim, algoritmaların daha hızlı doğrulanması ve iyileştirilmesi konusunda açık kaynaklı Motion Synthesis Araç Seti kullanılarak bu işlemlerin kolaylıkla yapıldığ görülmüştür (Bennett, Massey, Wu, Hasnain ve Jafari, 2016). Benzer bir başka çalışmada, bir kullanıcının sağlığı ile ilgili klinik olarak anlamlı bilgilerin kaydedilmesi için bir biyopotansiyel veri kaydedici tasarlanmış olup cihazın test edilmesi sonucunda elektrokardiyograf (EKG) ve ivme ölçer sinyallerinin alındığ doğrulanmıştır (Cook, Gargiulo, Lehmann ve Hamilton, 2015). İnsan kalbinin elektriksel aktivitesini tek bir kanaldan yakalayabilen ve gösterebilen bir EKG'nin yapısı bu çalışmada modellenmiştir (Costa Martinez, Walter Dafico Pfrimer, Scroccaro Costa ve Yoshihiro Nakano, 2018).

Spesifik sağlık alanlarının dışında, sağlık hizmetlerinde Açık Kaynak Elektronik Tıbbi Kayıtlar yaygın olarak kullanılmaktadır. $\mathrm{Bu}$ sistemde iş akışındaki aksaklığı, sistemin analizini ve keşfini kontrol etmek için Süreç Madenciliği kullanılmıştır. Uygunluk kontrolü sonuçları, Açık Kaynak EMR'deki süreçlerin hastaneler tarafindan yürütülen süreçlerle uyumlu olduğunu göstermiştir (Asare, Wang ve Fang, 2020). Sağlık alanında ele alınan açık kaynak konulu makalelerden elde edilen sonuçlar, sağlık alanında modelleme kolaylığı ve kullanılabilirliğin sağladığı avantajlar ile deneylerin ve görselleştirme işlemlerinin daha kolay yapılabileceği yönünde olmuştur.

\subsection{Teknoloji}

Özgür-Açık Kaynak Yazılımlar ve Donanımlar son yıllarda karşımıza her alanda sıklıkla çıkmaya başlamıştır. FLOSS teknolojisi ağ güvenliği, haberleşme, robotik, yapay zeka ve görüntü işleme gibi çeşitli alanlarda kullanılmaktadır. Görüntü işleme alanına dahil edebileceğimiz bir çalışmada; doğal bir göldeki balık türlerini araştırmak için balık türlerini tanımaya odaklanılmıştır ve drone'un gözü görevi gören 360 panoramik kameralı bir sualtı drone geliştirilmiştir. Yapılan çalışmalar neticesinde, neredeyse tüm balık türlerinin Alex-Net ve GoogLeNet ile \%85'in üzerinde bir tanıma oranıyla doğruluğu saptanmıştır (Meng, Hirayama ve Oyanagi, 2018).

Açık kaynak donanım kullanarak parmak izi eşleştirme çalışmaları da görüntü işleme alanına dahil edilebilir. $\mathrm{Bu}$ çalışmada, RaspiReader'ın FTIR çıktısından elde edilen görüntüler ile bir ticari kullanıma hazır(COST) okuyucudan alınan görüntüler arasındaki parmak izi eşleştirme deneyleri, Raspi-Reader'ın mevcut COTS optik okuyucularla birlikte önceki yöntemler göz önünde bulundurularak belirgin bir performans artışı sağladığı kanısına varılmıştır (Engelsma, Cao ve Jain, 2019).

Teknolojik alan içerisinde açık kaynak kullanımı robotik alanda karşımıza sıklıkla çıkmaktadır. Robotik alanını konu alan makalede, robotların kontrolü esnasında motor kontrolörü için açık kaynaklı bir Vedder elektronik hız kontrolörü (VESC) kullanılmıştır. Mevcut hız ve konum kontrol performanslarını değerlendirmek için MJ-VESC, bir 3D yazıcı ile üretilebilen açık kaynaklı bir planet dişli aktüatöre uygulanmıştır. Sonuç olarak, aktüatör modülünün bir 3D yazıcı kullanılarak düşük maliyetle üretilmesine rağmen, yüksek tork verimliliği ve tekrarlanabilirliğin elde edilebileceği gösterilmiştir (Choi, 2020).

Açık kaynaklı yazılım ve açık donanım kullanarak akıllı e-bisiklet izleme sistemi (SEMS) tasarlamak için, sistemden anlık veri toplamak ve toplanan verileri incelemek için projeye açık kaynak küresel konumlama sistemi (GPS) sensörü dahil edilip anlık bilgilere erişilmiştir. Tasarlanan sistem uzun süre boyunca kararlı bir şekilde çalıştırılıp ve aynı anda kullanılan çok sayıda e-bisiklet sistemi ile önceden belirlenen tasarım hedeflerinin yerine getirilmesi sağlanmıştır (Kiefer ve Behrendt, 2016).

Haberleşme sistemlerinde, afet dönemlerinde kritik seslerin daha iyi tespit edilmesi adına sistemlerin protokol bant yapılarının incelemesi yapılmıştır (Kumbhar, Koohifar, Güvenç ve Mueller, 2017). Çalışmada NS-3 açık kaynak aracı kullanılarak bir simülasyon çalışması yapılmış olup uzun vadeli erişim (LTE) bant sınıfının LMRS abone birimiyle karşılaştırıldığında önemli ölçüde daha yüksek verim elde edildiği görülmüştür. FLOSS yapısında hataların hızlı düzeltilmesi büyük bir avantaj sağlamaktadır. Yapılan bir çalışmada, çeşitli sistemler üzerindeki güvenlik açıklarını önlemek adına bir tarama testi önerilmiştir ve bu yöntem, hızlı bir şekilde tahmin etmeye dayalı otomatik bir yapıdır. Çalışma 
sonucunda, testin güvenlik açıklarının tespitinde yararlı olabileceği ileri sürülmüştür (Dashevskyi, Brucker ve Massacci, 2019).

Açık kaynak yazılımların hızla gelişmesindeki temel etkenlerden biri de kaynakların açık olup bunlarda meydana gelecek en ufak bir hatanın hızlı tespiti ve düzeltilmesi imkanıdır. Bu makalede, FLOSS un son yıllardaki gelişimi için dönemsel gönüllülük (EV) kavramından bahsedilmiş ve dönemsel gönüllülük kavramının FLOSS'ta yaygın olduğu kanısına varılmıştır. Bir diğer geliştirici olan IoT geliştiricilerin, IoT dışı uygulamalardan nasıl farklılaştığını daha derin bir şekilde anlamak için Açık Kaynak Yazılım (OSS) bağlamında IoT uygulamalarına nasıl katkıda bulunduğu araştırılmıştır (Corno, De Russis ve Saenz, 2020). Çalışma sonucuna göre, IoT senaryosunda yazılım mühendisliği ihtiyaçlarını daha iyi karşılamayı amaçlayan, gelecekteki araştırma çabalarında dikkate alınacak bazı Açık Kaynak IoT yazılım geliştirme özellikleri hakkında kanıtlar sergilemektedir. Teknoloji alanında açık kaynak ile ilgili incelenen makalelerde genel anlamda donanımların kullanılabilirliği, maliyet avantajı ve güvenlik avantajlarından yararlanılırken; yazılım da ise geliştirilebilirlik, güvenlik ve uygulanabilirlik kavramlarının tercih sebepleri olduğu çıkarımı yapılabilir.

\section{Sonuç}

$\mathrm{Bu}$ makalede, açık kaynak ve özgür yazılımları benimseme kararını etkileyebilecek faktörler üzerinde durulmuş olup son yıllarda FLOSS un kullanım alanlarındaki değişimin analizi yapılmıştır. Sistematik bir literatür taraması yöntemi uygulanarak Açık Kaynak ve Özgür Yazılımlar başlığı altında çeşitli alanlarda makaleler ele alınarak incelemeler yapılmış ve bunlar kendi aralarında kategorize edilmiştir. Bu kategorizasyon çalışması, "Eğitim", "Sağlık", "Bilişim-Teknoloji" ve "Endüstri" olmak üzere alt başlıklar şeklinde ele alınmıştır. Çalışmalar incelendiğinde, maliyet, kullanılabilirlik ve güvenlik başlıkları kullanıcıların açık kaynağı tercih etmesindeki faktörler olarak belirlenmiştir. Açık kaynak donanımlarının maliyetinin düşük olmasından dolayı eğitim ve sağlık alanlarında kullanımın gün geçtikçe arttığı kanısına varılmıştır. Eğitim alanında üniversite öğrencilerinin düşük maliyetli donanım kitlerini temin ederek laboratuvar derslerine ek olarak çalışma ve kendilerini geliştirme imkanı bulmuşlardır. Sağlık alanında ise, çalışma imkanı zor olan konuların açık kaynak donanım ve yazılım ile modellemesi oluşturularak çalışma alanlarında büyük avantaj elde edildiği görülmüştür.Bunun yanı sıra son yıllarda açık kaynağın güvenlik hususunda sunduğu imkanlar dahilinde BT alanında ve diğer çeşitli kamu kurumlarında kullanımın arttığı görülmektedir. Kamuda meydana gelen bu artışın sebebi, açık kaynak yazılımlarından ortaya çıkan güvenlik açıklarının hızlı bir şekilde tespit edilip ve bunların giderilmesi olarak belirlenmiştir. Bu bilgiler neticesinde, değişimler değerlendirilerek son 8-10 yıl içerisindeki kullanımı hızla artmakta olan açık kaynak yazılımları ve donanımlarının önümüzdeki yıllarda Eğitim, Sağlık, Endüstri başta olmak üzere diğer alanlarda da bir artış yaşanacağı beklenmektedir.

\section{Kaynakça}

Acuna, F., Rivas, D., Chancusi, S. ve Navarrete, P. (2015). Design and construction of a 3D printer auto controller wirelessly through of free software. IEEE Latin America Transactions, 13(6), 1893-1898. doi:10.1109/TLA.2015.7164214

Al Faruque, M. A. ve Vatanparvar, K. (2016). Energy Managementas-a-Service over Fog Computing Platform. IEEE Internet of Things Journal, 3(2), 161-169. doi:10.1109/JIOT.2015.2471260

Anzalone, G. C., Zhang, C., Wijnen, B., Sanders, P. G. ve Pearce, J. M. (2013). A low-cost open-source metal 3-D printer. IEEE Access, 1, 803-810. doi:10.1109/ACCESS.2013.2293018

Asare, E., Wang, L. ve Fang, X. (2020). Conformance Checking:
Workflow of Hospitals and Workflow of Open-Source EMRs. IEEE Access, 8, 139546-139566. doi:10.1109/ACCESS.2020.3012147

Barcomb, A., Kaufmann, A., Riehle, D., Stol, K. J. ve Fitzgerald, B. (2018). Uncovering the Periphery: A Qualitative Survey of Episodic Volunteering in Free/Libre and Open Source Software Communities. IEEE Transactions on Software Engineering. doi:10.1109/TSE.2018.2872713

Bennett, T. R., Massey, H. C., Wu, J., Hasnain, S. A. ve Jafari, R. (2016). MotionSynthesis Toolset (MoST): An Open Source Tool and Data Set for Human Motion Data Synthesis and Validation. IEEE Sensors Journal, 16(13), 5365-5375. doi:10.1109/JSEN.2016.2562599

Cantürk, G. (2020). International Journal of Karamanoglu Mehmetbey Educational Research Etkileşimli Tahtalarda Açık Kaynak Kod Tabanlı Pardus İşletim Sisteminin Kullanımına Yönelik Öğretmen Görüşleri The Opinions of The Teachers on The Usage of Open Source Code Based Pardus, (July).

Chiesi, M., Guermandi, M., Placati, S., Scarselli, E. F. ve Guerrieri, R. (2019). Creamino: A Cost-Effective, Open-Source EEGBased BCI System. IEEE Transactions on Biomedical Engineering, 66(4), 900-909. doi:10.1109/TBME.2018.2863198

Choi, D. (2020). Development of Open-Source Motor Controller Framework for Robotic Applications. IEEE Access, 8, 14134 14145. doi:10.1109/ACCESS.2020.2965977

Cook, A. J., Gargiulo, G. D., Lehmann, T. ve Hamilton, T. J. (2015). Open platform, eight-channel, portable bio-potential and activity data logger for wearable medical device development. Electronics Letters, 51(21), 1641-1643. doi:10.1049/el.2015.2764

Corno, F., De Russis, L. ve Saenz, J. P. (2020). How is Open Source Software Development Different in Popular IoT Projects? IEEE Access, 8, 28337-28348. doi:10.1109/ACCESS.2020.2972364

Costa Martinez, A., Walter Dafico Pfrimer, F., Scroccaro Costa, M. ve Yoshihiro Nakano, A. (2018). How to Develop a Single Channel Electrocardiograph with a Low Budget. IEEE Latin America Transactions, 16(4), 1057-1063. doi:10.1109/TLA.2018.8362137

Dashevskyi, S., Brucker, A. D. ve Massacci, F. (2019). A Screening Test for Disclosed Vulnerabilities in FOSS Components. IEEE Transactions on Software Engineering, 45(10), 945-966. doi:10.1109/TSE.2018.2816033

Dawood, K. A., Sharif, K. Y., Zaidan, A. A., Abd Ghani, A. A., Zulzalil, H. B. ve Zaidan, B. B. (2019). Mapping and Analysis of Open Source Software (OSS) Usability for Sustainable OSS Product. IEEE Access, 7, 65913-65933. doi:10.1109/ACCESS.2019.2914368

DeKoenigsberg, G. (2008). How successful open source projects work, and how and why to introduce students to the open source world. Software Engineering Education Conference, Proceedings, 274-276. doi:10.1109/CSEET.2008.42

Dorodchi, M. ve Dehbozorgi, N. (2016). Utilizing open source software in teaching practice-based software engineering courses. Proceedings - Frontiers in Education Conference, FIE, 2016-Novem. doi:10.1109/FIE.2016.7757683

Engelsma, J. J., Cao, K. ve Jain, A. K. (2019). RaspiReader: Open Source Fingerprint Reader. IEEE Transactions on Pattern Analysis and Machine Intelligence, 41(10), 2511-2524. doi:10.1109/TPAMI.2018.2858764

Heradio, R., Chacon, J., Vargas, H., Galan, D., Saenz, J., De La Torre, L. ve Dormido, S. (2018). Open-Source Hardware in Education: A Systematic Mapping Study. IEEE Access, 6, 72094-72103. doi:10.1109/ACCESS.2018.2881929 
Jahn, I., Hohn, F., Chaffey, G. ve Norrga, S. (2020). An OpenSource Protection IED for Research and Education in Multiterminal HVDC Grids. IEEE Transactions on Power Systems, 35(4), 2949-2958. doi:10.1109/TPWRS.2020.2970477

Kapitsaki, G. M. ve Charalambous, G. (2016). Find your open source license now! Proceedings - Asia-Pacific Software Engineering Conference, APSEC, $0, \quad 1-8$. doi:10.1109/APSEC.2016.012

Kazman, R., Goldenson, D., Monarch, I., Nichols, W. ve Valetto, G. (2016). Evaluating the Effects of Architectural Documentation: A Case Study of a Large Scale Open Source Project. IEEE Transactions on Software Engineering, 42(3), 220-260. doi:10.1109/TSE.2015.2465387

Kiefer, C. ve Behrendt, F. (2016). Smart e-bike monitoring system: Real-time open source and open hardware GPS assistance and sensor data for electrically-assisted bicycles. IET Intelligent Transport Systems, 10(2), 79-88. doi:10.1049/ietits.2014.0251

Kumbhar, A., Koohifar, F., Güvenç, I. ve Mueller, B. (2017). A Survey on Legacy and Emerging Technologies for Public Safety Communications. IEEE Communications Surveys and Tutorials, 19(1), 97-124. doi:10.1109/COMST.2016.2612223

Lerner, J. ve Tirole, J. (2005). The scope of open source licensing. Journal of Law, Economics, and Organization, 21(1), 20-56. doi:10.1093/jleo/ewi002

Meng, L., Hirayama, T. ve Oyanagi, S. (2018). Underwater-Drone with Panoramic Camera for Automatic Fish Recognition Based on Deep Learning. IEEE Access, 6, 17880-17886. doi:10.1109/ACCESS.2018.2820326

Milla, A. C., Kurt, O. ve Mataruna-Dos-santos, L. J. (2019). User perceptions of technology integration in schools: Evidence from Turkey's fatih project. International Journal of Education and Practice, 7(4), 430-437. doi:10.18488/journal.61.2019.74.430.437

Pesek, M., Vucko, Z., Savli, P., Kavcic, A. ve Marolt, M. (2020). Troubadour: A gamified e-learning platform for ear training. IEEE Access, 8, 97090-97102. doi:10.1109/ACCESS.2020.2994389

Pisha, L., Garudadri, H., Warchall, J., Zubatiy, T., Hamilton, S., Lee, C. H., ... Rao, B. D. (2019). A Wearable, Extensible, OpenSource Platform for Hearing Healthcare Research. IEEE Access, 7, 162083-162101. doi:10.1109/ACCESS.2019.2951145

Rajagopal, A., Dembia, C. L., DeMers, M. S., Delp, D. D., Hicks, J. L. ve Delp, S. L. (2016). Full-Body Musculoskeletal Model for Muscle-Driven Simulation of Human Gait. IEEE Transactions on Biomedical Engineering, 63(10), 2068-2079. doi:10.1109/TBME.2016.2586891

Rodriguez-Sanchez, M. C., Torrado-Carvajal, A., Vaquero, J., Borromeo, S. ve Hernandez-Tamames, J. A. (2016). An Embedded Systems Course for Engineering Students Using Open-Source Platforms in Wireless Scenarios. IEEE Transactions on Education, 59(4), 248-254. doi:10.1109/TE.2016.2526676

Sánchez, V. R., Ayuso, P. N., Galindo, J. A. ve Benavides, D. (2020). Open Source Adoption Factors-A Systematic Literature Review. IEEE Access. Institute of Electrical and Electronics Engineers Inc. doi:10.1109/ACCESS.2020.2993248

Schmitz, A. ve Piovesan, D. (2016). Development of an OpenSource, Discrete Element Knee Model. IEEE Transactions on Biomedical Engineering, 63(10), 2056-2067. doi:10.1109/TBME.2016.2585926

Singh, V. B., Sharma, M. ve Pham, H. (2018). Entropy based software reliability analysis of multi-version open source software. IEEE Transactions on Software Engineering, 44(12), 1207-1223. doi:10.1109/TSE.2017.2766070

Wang, L., Wan, J. ve Gao, X. (2019). Toward the health measure for open source software ecosystem via projection pursuit and real-coded accelerated genetic. IEEE Access, 7, 87396-87409. doi:10.1109/ACCESS.2019.2926306

Yakimov, P. (2018). Towards Attractive STEM Education by Using Open Source Hardware and Software. 2018 IEEE 27th International Scientific Conference Electronics, ET 2018 Proceedings, 1-4. doi:10.1109/ET.2018.8549579

Zhang, X., Wang, X. ve Kang, Y. (2018). Change-oriented open source software process simulation. IEEE Access, 6, 7014570163. doi:10.1109/ACCESS.2018.2880998

Zhou, M. ve Mockus, A. (2015). Who Will Stay in the FLOSS Community? Modeling Participant's Initial Behavior. IEEE Transactions on Software Engineering, 41(1), 82-99. doi:10.1109/TSE.2014.2349496 